\title{
Post-Transplant Maintenance Therapy for Patients with Acute Myeloid Leukemia: Current Approaches and the Need for More Trials
}

This article was published in the following Dove Press journal: Journal of Blood Medicine

\author{
Rita Assi ${ }^{1}$ \\ Nohad Masri ${ }^{1}$ \\ Iman Abou Dalle ${ }^{2}$ \\ Jean El-Cheikh (D) ${ }^{2}$ \\ Ali Bazarbachi \\ 'Division of Hematology-Oncology, \\ Lebanese American University and \\ Lebanese American University Medical \\ Center-Rizk Hospital, Beirut, Lebanon; \\ ${ }^{2}$ Division of Hematology-Oncology, \\ American University of Beirut Medical \\ Center, Beirut, Lebanon
}

\begin{abstract}
Relapse rates following allogeneic stem cell transplantation for acute myeloid leukemia remain unacceptably high and a major cause of death. Maintenance therapies posttransplant administered either to patients with impending relapse or at high risk of relapse could present a strategy to improve survival and overall outcomes. With the increasing use of molecular and genomic characterization of the disease, more novel therapies became available as maintenance strategies. These options were, however, hindered by excessive toxicities, mostly hematologic, especially with the use of myeloablative conditioning regimens. Several key questions have also emerged including the efficacy of these therapies, the duration of maintenance, as well as the potential modulation of the graft and the immune microenvironment. These issues are further complicated by the paucity of well-designed prospective randomized clinical trials evaluating these agents. Future directions in this field should include better risk stratification and patient selection based on assays of minimal residual disease, as well as the incorporation of novel targets and pathways of leukemogenesis. In this article, we highlight the current evidence behind the use of post-transplant maintenance therapy, the optimal patient and disease selection, as well as the challenges faced by these strategies in an area that remains quite controversial. We will focus on therapies targeting leukemia stem cells that directly or indirectly modulate the allografted immune microenvironment and augment the graft-versus-leukemia impact.
\end{abstract}

Keywords: AML, maintenance, relapse, target, MRD

\section{Background}

Acute myeloid leukemia (AML) remains the most common acute leukemia in adults with an incidence of 3-4 per 100,000 person per year. AML is a genetically and phenotypically heterogeneous and biologically dynamic spectrum of diseases. ${ }^{1}$ Indeed, the clinical outcomes are largely determined by the patient's characteristics such as age, performance status and comoridities, as well as the leukemia features including the subtype (de novo versus secondary) and most importantly the genomic profile. ${ }^{2}$ The recent advances in defining the molecular landscape of AML and its role in leukemogenesis have paved the way for the development and adaptation of novel targeted agents.

Following induction chemotherapy, patients achieving a morphologic leukemiafree state (complete remission (CR)) are mandated to receive a form of consolidation therapy aimed at the residual leukemic stem cells (LSCs) to prevent relapse and improve overall survival (OS). ${ }^{3}$ A risk-adapted approach for relatively young or fit
Correspondence: Ali Bazarbachi Department of Internal Medicine, American University of Beirut, Medical Center, P.O. Box II 3-6044, Beirut, Lebanon

Email bazarbac@aub.edu.lb

Journal of Blood Medicine 2021:12 21-32 
AML patients in first CR (CR1) involves the assessment of this risk of relapse, leading to either chemotherapy continuation or allogeneic stem cell transplantation (ASCT), taking into account the presence of comorbidities, the donor type as well as the genetic characteristics of the disease. ${ }^{4}$ In addition to pre-treatment risk stratification, the estimation of the leukemic burden while on therapy has recently emerged as a strong, independent and dynamic tool for individualizing post-induction treatment approaches. Either polymerase chain reaction (PCR), multiparameter flow cytometry (MFC) or the novel nextgeneration sequencing (NGS) can evaluate this measurable residual disease $(\mathrm{MRD})^{5-7}$

\section{Allogeneic Stem Cell Transplantation: Rationale and Nuances}

Up to the current date, ASCT in first CR remains the most powerful antileukemic post-remission therapy. ASCT is generally recommended upfront for properly selected patients with high-risk cytogenetic features, those with intermediate and adverse-risk molecular findings, and patients with secondary AML. Patients with induction failure, post-induction residual disease and following salvage therapy are also referred for ASCT. In addition to potentially life-threatening complications of ASCT such as graft-versus-host disease (GVHD) and opportunistic infections, survival benefits recorded with ASCT are crippled by unacceptably high disease relapse rates, ${ }^{8-10}$ hence the need for strategies to maintain remission and prevent relapses post-ASCT. Such interventions aim at reinforcing the graft-versus-leukemia (GVL) effect and/or eradicating persistent MRD, especially with the increasing availability of more sensitive techniques to detect any residual disease. Nevertheless, these maintenance therapies may represent over-treatment for patients with intermediate-risk disease, further subjecting them to long-term toxicities and disturbed quality of life (QoL), thereby reinforcing the need for a better selection of patients as well as strict and continuous MRD monitoring.

The transplantation field has tremendously evolved over the last two decades with refinements of indications as well as improvement in the safety profile of conditioning regimens and supportive care strategies. Nonetheless, risk factors for increasing mortality after relapse in an allografted patient still include, among others, a shorter time to recurrence and occurrence of GVHD prior to relapse ${ }^{11}$ with significant improvement of overall survival (OS) for young patients relapsing in recent years (Bazarbachi et al, 2020). ${ }^{12}$ Furthermore, a deeper understanding of factors facilitating disease relapse, such as molecular profile and role of MRD, has enabled more high-risk patients to receive post-transplant therapies to treat and even prevent relapses. Indeed, pharmacological intervention and manipulation of the disease kinetics in the early post-transplant phase could potentially collaborate with other strategies to improve overall outcomes, ${ }^{13}$ possibly through up-regulation of tumor-associated antigens (TAA),${ }^{14}$ expansion of regulatory T-cells,,${ }^{15}$ or acceleration of T-cell reconstitution. ${ }^{16}$ With the availability of a wide array of novel and less toxic agents such as epigenetic modifiers, tyrosine kinase inhibitors (TKIs), BCL2 inhibitors and immune checkpoint inhibitors (ICPIs) among others, an intriguing strategy would be to preemptively use such molecules in an attempt to prevent relapses postASCT in specific subsets of high-risk patients. Nevertheless, we currently only have few randomized trials that offered a survival advantage for maintenance therapy in AML.

\section{Selection of Optimal Candidates}

Conducting either retrospective studies or prospective randomized trials to construct therapeutic strategies aiming at reducing post-ASCT relapse rates has been historically hampered by the depth of remission achieved as well as the intrinsic biologic apparatus of the disease. Cytogenetic abnormalities of AML knowingly dictate both the general outcomes of standard therapies and those following ASCT. ${ }^{17}$ In view of the granular advances in the field of myeloid malignancies, considering specific subsets of AML patients for post-ASCT maintenance should therefore depend on the molecular and genomic characteristics of the disease itself at diagnosis. ${ }^{18}$ Indeed, the presence of actionable or targetable mutations such as FLT3-ITD and $I D H 1 / 2$ is a valuable opportunity to incorporate the approved corresponding inhibitors in the post-ASCT maintenance strategies. Novel molecular and MRD diagnostics are therefore of utmost importance to determine those who would benefit the most from personalized therapy options. As such, MRD status in the pre-transplant phase and more importantly detection of MRD early post-ASCT are crucial factors to implement therapy as they largely impact the likelihood and pace of disease relapse. ${ }^{19,20}$

In this setting, other variables including the donor source, intensity of conditioning regimen and GVHD 
prophylaxis protocols (T-cell depletion and post-ASCT cyclophosphamide) might influence the risk of disease relapse. ${ }^{21}$ While the implementation of reduced-intensity conditioning (RIC) has allowed more patients to receive $\mathrm{ASCT}^{22}$ it could potentially increase the rate of posttransplant relapse, as demonstrated by the large prospective randomized Phase III trial conducted by the Bone Marrow Transplant Clinical Trials Network. ${ }^{23}$ Welldesigned trials are eagerly needed to appropriately answer these challenging situations.

\section{Challenges to Conduct Clinical Trials}

In the presence of few prospective randomized trials, the decision to initiate post-ASCT maintenance therapy remains ambivalent in many situations. Early-phase studies assessing novel agents in the relapsed setting often exclude patients with prior history of ASCT given the plethora of complications they might experience, therefore resorting to agents previously approved for different indications or settings. This dilemma largely provides a protective blanket to access these drugs on an off-label indication, which could impede recruitment for prospective studies. Additionally, most currently ongoing maintenance trials using hypomethylating agents (HMA), targeted therapies and other molecules still demand rigorous eligibility criteria, thereby interfering with enrollment rate.

\section{Optimal Timing and Duration of Maintenance Initiation}

Starting maintenance therapy in the early post-ASCT phase should take into account the concomitant use of immunosuppressive drugs and their potential heightened hematological and organ toxicities, the risk of opportunistic infections and GVHD, as well as the possible drugdrug interactions (such as with calcineurin inhibitors), even when the acute toxicities of ASCT have seemingly resolved. An optimal maintenance approach is therefore difficult to be intercalated within the conditioning regimen itself and is reserved for a post-ASCT phase, mostly started between days 30 and 100 following transplantation. In this setting, pre- and post-ASCT MRD status could be valuable in planning and timing maintenance therapy. For those patients with impending signs of relapse by MRD testing or falling donor chimerism, a preemptive maintenance therapy could be started early post-ASCT, before overt morphological relapse.
Finally, the optimal duration of maintenance therapy has not been established for most cases, thereby affecting the QoL of these patients.

\section{Available Maintenance Options: The Current Evidence Targeting Epigenetics Pathways of Leukemia Cells Hypomethylating Agents}

The use of HMAs such as azacitidine and decitabine remains the most commonly adopted non-targeted strategy for the prevention of post-ASCT relapse owing in part to their acceptable safety profile. ${ }^{24}$ The mechanism of action of HMAs post-ASCT is unclear, but they appear to silence tumor suppressor genes through epigenetic modification. At the preclinical level, these agents could also induce a GVL effect through stimulation of CD8+ T-cell responses to overexpressed tumor-associated antigens (TAAs) such as MAGE antigens. ${ }^{25}$ This activity has led to the investigation of HMAs in a series of small trials, especially with the advancing field of MRD detection by sensitive techniques.

For example, AML patients with imminent relapse due to decreasing CD34 chimerism received pre-emptive azacitidine that delayed disease progression according to two studies. ${ }^{26,27}$ The concurrent administration of donor lymphocyte infusion (DLI) did not, however, improve response rates or $\mathrm{OS}^{27}$ and the majority of patients eventually experienced overt disease relapse. ${ }^{26}$ In another study, azacitidine was also given sequentially with DLI and showed a low relapse rate and encouraging OS despite the presence of acute and chronic GVHD. ${ }^{28}$

In a Phase I dose-finding trial, azacitidine as monotherapy was given between on day +42 post-ASCT to 45 patients with AML (82\%) and MDS, for up to four cycles at different dose levels $8,16,24,32$, and $40 \mathrm{mg} / \mathrm{m}^{2}{ }^{29}$ Interestingly, two-thirds of AML patients were not in CR at the time of transplant. The recommended dose of azacitidine was reported to be $32 \mathrm{mg} / \mathrm{m} 2$ for 5 days in 30-day cycles because of dose-limiting but reversible thrombocytopenia. At 1-year follow-up, the median disease-free survival (DFS) was 58\% for all enrolled patients and the 1-year OS rate was $77 \%$. In another phase I/II study of 27 AML patients who received a RIC regimen followed by ASCT later showed that the subcutaneous administration of up to 10 cycles of azacitidine at $36 \mathrm{mg} / \mathrm{m}^{2}$ for 5 days in 28-day cycles beginning at day 42 post-ASCT resulted in 
the expansion of circulating regulatory T-cells with subsequent GVL response and no significant GVHD. ${ }^{15}$ In a retrospective study of 18 allografted patients (13 AML and $5 \mathrm{MDS}$ ), including $50 \%$ of patients with a high or very high disease risk index, low-dose azacitidine started at a median of 60 days post-transplant was well tolerated and resulted in one-year disease-free survival (DFS) and OS of $63 \%$ and $70 \%$, respectively. ${ }^{30}$ A subsequent randomized phase III trial comparing azacitidine at $32 \mathrm{mg} / \mathrm{m} 2$ subcutaneously for 5 days in up to 12,28-day cycles to no intervention in 87 patients with AML, myelodysplastic syndromes (MDS) or chronic myelomonocytic leukemia in remission was terminated early because of slow accrual. ${ }^{31}$ At a median follow-up of 4.6 years in the azacitidine arm, available data suggest no significant effect of the HMA on relapse-free survival (RFS), except for a non-statistically significant trend for improvement in those who received at least 9 cycles of therapy.

The importance of MRD-adapted therapy is highlighted in the ongoing Phase II study (RELAZA2) whereby preemptive treatment with at least 6 cycles of azacitidine $\left(75 \mathrm{mg} / \mathrm{m}^{2} \times 7\right.$ days) and for up to 18 additional months was evaluated. ${ }^{32}$ The study enrolled patients in CR but with detectable MRD either after conventional chemotherapy or following ASCT. This preemptive MRD risk-adapted strategy was found to prevent or significantly delay disease relapse in $58 \%$ of patients who remained in CR after 6 months $(95 \%$ CI: 44-72; p $<0.001)$. These results are encouraging and warrant further follow-up.

More recently, an oral azacitidine formulation CC-486 with extended dosing to prolong activity of azacitidine with sustained DNA hypomethylation showed promising results as maintenance therapy in a randomized trial following induction chemotherapy for AML. ${ }^{33}$ CC-486 was then evaluated in a phase I/II trial of 30 patients (26 with AML and 4 MDS) who had undergone ASCT, given at 200-300 mg orally for 7 days or 150-200 mg orally for 14 days in up to 12,28 -day cycles. ${ }^{34}$ The study resulted in 1 -year RFS rates of 54\% with the 7-day protocol and $72 \%$ with the 14-day regimen in the 28 evaluable patients, leading to estimated 1-year survival rates of $86 \%$ and $81 \%$, respectively. The most common grade 3-4 treatment-related toxicities were gastrointestinal and hematologic toxicities, and two patients experienced severe chronic GVHD. A randomized, phase III trial evaluating CC-486 at the $200 \mathrm{mg}$ 14-day dosing regimen as maintenance therapy post-ASCT for high-risk MDS and intermediateor high-risk AML is currently enrolling.
On the other hand, a small study of decitabine administered at $5-15 \mathrm{mg} / \mathrm{m} 2$ intravenously for 5 days starting 50-100 days post ASCT for up to 8, 6-week cycles also exhibited favorable results with 2-year OS of $56 \%$ and cumulative incidence of relapse reaching $28 \%{ }^{35}$ However, the majority (75\%) of patients experienced grade 3-4 hematologic toxicities during therapy. While decitabine did not increase the rate of chronic GVHD, there was a trend for increased FOXP3 expression and T-reg cells in the lymphocyte environment in a correlative study that was not statistically meaningful.

Interpreting the results of these studies remains challenging and controversial, as they are small and mostly uncontrolled. As such, the optimal timing of HMA initiation post-ASCT and dosing need to be explored further to establish efficacy at preventing relapses and avoid unnecessary toxicities, especially in patients who can be cured with ASCT alone. In patients with detectable MRD or mixed chimerism, pre-emptive treatment with HMA could potentially delay or even prevent relapses in AML and MDS patients. ${ }^{36}$

More recently, there has been a growing interest in evaluating HMA as partners to novel promising agents such as the BCL2 inhibitor venetoclax, ICPs, FLT3 inhibitors, as well as isocitrate dehydrogenase (IDH) inhibitors and studies are ongoing (Table 1).

\section{Histone Deacetylase Inhibitors (HDACi)}

The class I/II HDACi have presented as potential promising agents in AML/MDS owing to large induction effects on cell-cycle arrest and differentiation, as well as proapoptotic effects on myeloid cells through epigenetic modifications of histones. ${ }^{37} \mathrm{HDACi}$ have also exhibited some antileukemic and immunomodulatory roles through the control of cytokine secretion. This is further evidenced by the panobinostat activity, a potent oral inhibitor of class 1,2 , and 4 deacetylases, in the PANOBEST trial. ${ }^{38}$ This study enrolled 42 patients with high-risk AML or MDS who had received ASCT and panobinostat was started at a median of 98 days (60-150) post-ASCT. Twothirds of these patients were transplanted in active disease. While only $22(54 \%)$ of the 42 patients completed 1 year of therapy because of adverse events, the cumulative incidence at relapse remained $21 \%$ at 2 years, resulting in 2 -year OS and DFS rates of $88 \%$ and $74 \%$. More importantly, panobinostat was found to inhibit the suppressive function of T-regs when used at low doses and enhance their function at higher doses, ${ }^{39}$ thereby playing a possible 
Table I Some of the Ongoing Trials Evaluating Various Targets for Post-Allogeneic Stem Cell Transplantation Strategies

\begin{tabular}{|c|c|c|c|c|c|}
\hline Target & Drug(s) & Clinical Phase & Endpoints & Status & Identifier \\
\hline \multirow[t]{3}{*}{ FLT3 } & & & & & \\
\hline & Crenolanib & II & PFS; DFS; OS; GVHD; I00-day TRM & Recruiting & NCT02400255 \\
\hline & Gilteritinib & III & RFS, OS, GVHD, EFS & Active, not recruiting & NCT02997202 \\
\hline \multirow[t]{2}{*}{ TP53 } & & & & & \\
\hline & APR-246 + Azacytidine & II & Safety, tolerability, I-year RFS & Recruiting & NCT0393 I29I \\
\hline \multirow[t]{2}{*}{ Hh } & & & & & \\
\hline & Glasdegib & III & DFS & Not yet recruiting & NCT04I68502 \\
\hline \multirow[t]{5}{*}{ DNMT } & & & & & \\
\hline & Low-dose azacytidine & II & OS; relapse rate; safety & Recruiting & NCT0।995578 \\
\hline & Azacytidine+ valproic acid & II & OS; time to relapse & Recruiting & NCT02I24I74 \\
\hline & Oral azacytidine (AMADEUS) & III & RFS, OS, NRM, GVHD & Recruiting & NCT04I73533 \\
\hline & SGI-II0+ DLI & II & DFS, OS & Not yet recruiting & NCT03454984 \\
\hline \multirow[t]{4}{*}{ BCL-2 } & & & & & \\
\hline & Venetoclax + azacytidine & I & MTD; OS, RFS; GVHD & Recruiting & NCT036I3532 \\
\hline & Venetoclax + azacytidine & II & RFS, OS, GVHD & Recruiting & NCT04I2850I \\
\hline & Venetoclax+ azacytidine (VIALE-T) & III & RFS, OS, GVHD, MRD & Recruiting & NCT04I6I885 \\
\hline \multirow[t]{2}{*}{ HDAC } & & & & & \\
\hline & Panobinostat & III & OS, DFS, EFS, NRM, GVHD & Recruiting & NCT04326764 \\
\hline \multirow[t]{4}{*}{ IDH2 } & & & & & \\
\hline & Enasidenib & I & Safety, tolerability, GVHD, RFS & Recruiting & NCT03728335 \\
\hline & Enasidenib & I & MTD, DLT, GVHD & Recruiting & NCT035I55I2 \\
\hline & Enasidenib & II & OS, RFS, Safety and tolerability & Recruiting & NCT04522895 \\
\hline \multirow[t]{2}{*}{ IL-I5 } & & & & & \\
\hline & $\mathrm{N}-803$ & II & Relapse rate, GVHD, MRD, OS & Recruiting & NCT02989844 \\
\hline
\end{tabular}

Abbreviations: PFS, progression-free survival; OS, overall survival; RFS, relapse-free survival; EFS, event-free survival; GVHD, graft-versus-host disease; MTD, maximum tolerated dose; DLT, dose-limiting toxicity; DLI, donor lymphocyte infusion; MRD, minimal residual disease; NRM, non-relapse mortality; Hh, hedgehog pathway; DNMT, DNA methyltransferase; HDAC, histone deacetylase.

role in reducing GVHD. As these results are intriguing, a randomized multicenter phase III trial is currently comparing panobinostat $20 \mathrm{mg}$ orally three times weekly every second week to the standard of care as maintenance post-ASCT. Vorinostat, another HDACi, is also being combined with low-dose azacytidine for post-ASCT in a currently ongoing phase I dose-escalation clinical trial.

\section{Targeting Oncogenic Pathways of Leukemia Stem Cells \\ FLT3 Inhibitors}

Treatment of FLT3-ITD mutated AML remains challenging due to significant relapse rates and short remissions with available therapies despite the common historical use of ASCT in first CR. ${ }^{40}$ Nevertheless, FLT3-mutated AML is a heterogeneous disease that entails diversity in the type of FLT3 mutations and their insertion site, the FLT3-ITD allelic burden, and the presence of concurrent mutations; observations that further complicated the decision to proceed to ASCT in the first CR when feasible. ${ }^{41-43}$ This controversy is evidenced by the European LeukemiaNet guidelines suggesting, with some controversy, that ASCT should not be offered to patients with low-mutant allelic ratio. ${ }^{44-46}$ EBMT guidelines allowed ASCT in this setting and recommended it for all patients with FLT3-mutated AML (Bazarbachi et al, 2020). ${ }^{47}$

As such, the use of multi-kinase inhibitors of various generations has led to improved outcomes and achievement of deeper responses in FLT3-mutated AML. These TKIs, together with the incorporation of MRD assessment, have enabled the installation of post-transplant therapeutic strategies, ${ }^{48}$ as the 1-year OS of patients who relapse postASCT drops to less than 20\%. ${ }^{11}$ (Bazarbachi et al, 2020). ${ }^{12}$

The enthusiasm of using FLT3 TKIs stems not only from their direct cytotoxic properties but also involve an 
immunomodulatory effect synergizing with allografted T-cells. Several murine models have shown that sorafenib enhances the production of interleukin-15 (IL-15) production by leukemic cells, thereby promoting GVL effect. ${ }^{16}$ The same experiment showed that sorafenib reduced the activating transcription factor (ATF4) expression in leukemic cells, a negative regulator of IRF-7 interferon regulatory factor-7 (IRF-7) activation, which further enhances IL-15 transcription when activated. The exact mechanisms of FLT3 TKIs immunogenicity remain to be elucidated.

One of the earliest and most promising post-transplant maintenance approaches has been the administration of FLT3 inhibitors, limited to date to FLT3-ITD mutated AML patients. Despite multiple retrospective and prospective randomized trials evaluating the efficacy and safety of the use of FLT3 inhibitors as post-transplant maintenance, there is still a debate on the best agent to be used (off-label use of sorafenib versus potent second-generation FLT3 inhibitors), dosing and time of initiation. A consensus by the EBMT Acute Leukemia Working Party recommended the use of sorafenib $400 \mathrm{mg}$ twice daily in the posttransplant setting in the absence of active GVHD based on available data (Bazarbachi et al, 2020). ${ }^{47}$ Previous retrospective studies have demonstrated a lower risk of disease relapse following ASCT in patients with FLT3 ITD mutated AML who received post-transplant sorafenib maintenance (Antar, et al, 2014). ${ }^{49-53}$

In a phase I study involving 22 patients with FLT3-ITD AML receiving sorafenib maintenance post-ASCT, PFS at 1 year was $85 \%$ and OS was $95 \%{ }^{54}$ Encouraging results were subsequently reported in other small trials of sorafenib maintenance compared to historical controls, showing markedly lower relapse rates, improved RFS and relatively tolerable toxicities, while not significantly affecting the rates of GVHD. ${ }^{51-53,55-57}$ This is further supported by two registry studies from the European Society for Blood and Marrow Transplantation (EBMT) showing that posttransplant maintenance with sorafenib improved OS and leukemia-free survival (LFS) of allografted patients with FLT3-ITD positive AML (Bazarbachi et al, 2019) ${ }^{58}$ and that sorafenib combined with DLI clearly improved OS and LFS of relapsed FLT3-ITD positive AML patients following ASCT. (Bazarbachi et al, 2019) ${ }^{59}$

In a prospective phase II controlled randomized trial (SORMAIN) of 83 patients with FLT3-ITD mutated AML, the administration of sorafenib for up to 24 months resulted in superior outcomes for patients in $\mathrm{CR}$ and no grade $\geq 2$ GVHD compared to placebo. After a long median follow-up of 42 months, the 2-year RFS was $85 \%$ in the sorafenib group compared with $53 \%$ in the placebo group $(\mathrm{HR}=0.39, \mathrm{p}=0.01)$, in addition to an $\mathrm{OS}$ benefit for the sorafenib group $(\mathrm{HR}=0.447 ; \mathrm{p}=0.03){ }^{60}$ Further follow-up showed that many patients will experience disease relapse when sorafenib is stopped at 24 months, suggesting a longer exposure to sorafenib might be needed to prevent late relapses. While SORMAIN trial constitutes the first placebo-controlled evidence that postHSCT maintenance therapy could reduce the risk of relapse and death, this study enrolled patients who underwent transplantation in the first hematological CR, as well as those in the second or subsequent CR. Finally, the Chinese open-label, large randomized phase III trial assigned patients to receive sorafenib maintenance $(n=100)$ or control $(n=102)$ post-ASCT (Xuan et al 2020). ${ }^{61}$ At a median follow-up of 21.3 months, the 1-year cumulative incidence of relapse was 7.0\% $(95 \%$ CI 3.1-13.1) in the sorafenib group and 24.5\% (16.6-33.2) in the control group (hazard ratio $0.25,95 \%$ CI $0.11-0.57$; $\mathrm{p}=0.0010$ ), with no treatment-related deaths and acceptable GVHD rates. Based on these available data, sorafenib is recommended by many authorities as a maintenance strategy to reduce post-ASCT relapses for FLT3-ITDmutated AML (Bazarbachi et al, 2020). ${ }^{47}$

More recent data from the RATIFY trial that led to the US Food and Drug Administration (FDA) approval of midostaurin in 2017, proposed that the outcomes of patients who received this agent prior to ASCT were particularly encouraging. ${ }^{62}$ In a phase II trial of midostaurin received as post-consolidation or post-ASCT maintenance, the 1-year relapse rate was encouragingly low at 9.2\%. ${ }^{63}$ In this German-Austrian AML Study Group 16-10, most patients discontinued midostaurin earlier than planned because of toxicities. This remains in line with prior reports on the drug's complex pharmacokinetic profile and drug-drug interactions that warrant close observation and dose adjustments to reduce toxicity. ${ }^{64,65}$

RADIUS is another phase II randomized study that accrued 60 patients with FLT3-ITD AML with stable engraftment post-ASCT to receive or not midostaurin for twelve 4 -week cycles. ${ }^{66}$ Unsurprisingly, the median RFS was not reached for either arm as the trial was not powered to detect any statistical difference $(p=0.34)$ between subgroups.

The prospective cooperative group international phase III randomized trial (BMT-CTN 1506; NCT02997202) is seeking to confirm the impact of post-transplant gilteritinib 
maintenance therapy versus placebo in patients with FLT3mutated AML and has completed accrual at 346 patients. Gilteritinib is an effective and tolerable FLT3 inhibitor, with potent activity against both FLT3-ITD and FLT3TKD mutations, particularly the kinase domain mutations at residue D835 and the gatekeeper mutation at residue F691. ${ }^{67}$ Gilteritinib was recently approved for use in the relapsed/refractory setting ${ }^{68}$ and was chosen for evaluation as post-ASCT maintenance owing to its safety profile and potent inhibition of FLT3 in vivo. Unfortunately, the use of placebo as control arm in this trial will not allow to answer the important question of whether Gilteritinib offers an additional benefit over sorafenib in that setting.

Quizartinib (AC220), a highly potent selective FLT3ITD inhibitor was also studied in one small phase I trial where only 1 of 13 patients relapsed under therapy at the last follow-up. ${ }^{69}$ Furthermore, toxicities were manageable and GVHD rate was not increased. However, increasing reports about resistance through point-mutant forms have been emerging, hence limiting single-agent use. ${ }^{70}$

Crenolanib, like gilteritinib, is another potent oral type 1 FLT3 TKI with extended activity against FLT3-ITD and resistance-conferring FLT3-D835 TKD mutants. ${ }^{71}$ It is also under evaluation as a post-ASCT maintenance in a phase II trial (NCT02400255), in a cohort of patients transplanted in $\mathrm{CR}$ and in another group allografted with the residual disease with $\leq 10 \%$ bone marrow blasts. Crenolanib is started between days 45 to 90 after ASCT and for up to 2 years. It is important to note that phase II/ III trials of post-ASCT maintenance involving the novel FLT3 TKIs do not use a first-generation inhibitor control, making it difficult to establish their superior efficacy in this setting.

Some unanswered questions remain regarding the use of FLT3 TKIs as maintenance post-ASCT. FLT3-ITD mutations, unlike $B C R-A B L 1$ fusions, ${ }^{72}$ are not founding mutations but rather an important final step and one of many mutations found in leukemogenesis. ${ }^{73,74}$ These include $W T 1, I D H 1, D N M T 3 A$, as well as NUP98/NSD1 fusions, which are currently known to affect outcomes and response to therapy. Furthermore, FLT3 measuring assays are not cross-validated within trials along with considerable variability in the FLT3-ITD cut-off used ( 0.5 in the ELN recommendations, 0.7 in the RATIFY study) for treatment, as well as the dynamic changes that happen to this ratio over time. Until standardization of definitions, the indication of ASCT remains itself controversial in patients with low $(<0.5)$ allelic ratio FLT3-ITD who have a concomitant NPM1 mutation and achieve MRD negative status on therapy (Bazarbachi et al, 2020). ${ }^{47}$

\section{IDHI/IDH2 Inhibitors}

Ivosidenib and enasidenib have been recently approved for the treatment of IDH1 and IDH2-mutated AML, respectively. ${ }^{75,76}$ Owing to the natural history of this subtype of AML and the relative safety of these agents, they could present as a promising option for maintenance therapy postASCT. Some trials (NCT03515512, NCT03564821) are currently evaluating the significance of these mutations and their role in post-ASCT relapses, as well as the safety of the corresponding targeted agents in this setting.

\section{BCL2 Inhibitors}

Venetoclax is a BCL2 inhibitor that competitively binds to the $\mathrm{BH} 3$ domain of BCL2, an anti-apoptotic protein, releases $\mathrm{BH} 3$-only proteins and induces apoptosis of hematologic malignant cells. ${ }^{77}$ Venetoclax has been evaluated and is currently approved in combination with lowdose cytarabine and azacitidine or decitabine. ${ }^{78,79}$ These studies have included only a few patients who relapsed after ASCT and still achieved CR with the combination. Two prospective trials investigating the efficacy of venetoclax in combination with azacitidine at improving RFS are currently enrolling AML patients for maintenance or preemptive therapy post-ASCT.

\section{Hedgehog ( $\mathrm{Hh})$ Pathway Signaling Inhibitors}

Anomalous hedgehog (Hh) pathway signaling is involved in the survival and proliferation of leukemia stem cells, ${ }^{80}$ especially those resistant to chemotherapy. ${ }^{81}$ Glasdegib, an oral small Hh inhibitor, has been recently FDA approved in combination with low-dose cytarabine for the treatment of AML patients not eligible for intensive therapy, after showing OS benefit. ${ }^{82}$ Based on these findings, glasdegib is currently being evaluated in a phase II study for postASCT maintenance for AML patients at high-risk of relapse (NCT01841333).

\section{Agents Targeting Mutated P53}

AML and MDS with abnormal 17p or mutated p53 are known to portend dismal outcomes with the highest risk of relapse even in the post-ASCT phase. ${ }^{83}$ APR-246 is an agent that targets $p 53$ mutation in an attempt to restore its function and showed up to $80 \% \mathrm{CR}$ rate in an early trial of patients with myeloid malignancies. ${ }^{84}$ Based on this concept, a phase II trial studying the combination of azacytidine and APR-246 is currently enrolling allografted patients with MDS and 
AML and mutated p53 (NCT03931291) with a primary endpoint being 1-year RFS.

\section{Targeting Leukemic Surface Receptors Monoclonal Antibodies}

The use of antibody-drug conjugates (ADC) could achieve target specificity through inhibition of certain surface markers, such as CD33, expressed on the majority of myeloblasts. Gemtuzumab ozogamicin (GO) is a MoAb against CD33 conjugated to the toxin calicheamicin. In a small study of 10 relatively young patients allografted for highrisk AML, GO was administered with azacitidine as maintenance post-ASCT. ${ }^{85}$ After a median number of 1.5 cycles only complicated by reversible hematological toxicities, $40 \%$ of patients relapsed.

Another newer generation anti-CD33 ADC Vadastuximab talirine (SGN33a) conjugated to a pyrrolobenzodiazepine dimer was studied as maintenance in the post-ASCT setting (NCT02326584), but the phase I/II trial was terminated early because of neutropenia and thrombocytopenia.

\section{Immune-Mediated Therapies}

Maintenance therapy with immune checkpoint inhibitors, such as nivolumab, is being investigated in clinical trials for patients with high-risk AML in remission postconsolidation, who are not candidates for ASCT. ${ }^{86}$ For instance, using this selective immune modulation for postASCT maintenance may provide similar benefits and merits investigation owing to their inherent activity in AML. Nonetheless, issues related to acute GVHD are likely to emerge, as seen with previous studies of lenalidomide in this setting, ${ }^{87}$ thereby limiting the wide adoption of these agents. $^{88-90}$

Other agents on the outlook in this setting include antichemokine (C-X-C motif) receptor 4 (CXCR4) as well as CAR T-cell therapy.

\section{Our Personalized Approach for Post-ASCT Maintenance}

AML has increasingly presented itself as a poster child for personalized treatment approaches. ASCT by itself should not be regarded as an ultimate definitive therapy for all patients and with established poor outcomes for postASCT relapses, preventing one remains more beneficial than treating it. Nonetheless, we still have no simple algorithm or strategy to address post-ASCT relapses or maintenance approaches. As delineated above, most available information is derived from phase II trials of HMAs and FTL3-ITD TKIs and few randomized data. Recent development of targeted agents made their use in the posttransplant setting more exciting taking into consideration the potential risks on GVHD and immune reconstitution post-ASCT. Furthermore, better MRD assessments facilitated the optimal selection of high-risk candidates who would benefit from such strategies.

Any treatment decision should therefore involve the patient's performance status, the pre-transplant disease course, the presence of actionable mutations, and the use of concurrent immunosuppressive medications as well as GVHD. Prognostication of high-risk AML patients has been recently refined, especially with the introduction of various MRD assays. These include $\mathrm{MFC}^{5,91}$ and NGSMRD monitoring, both shown to be predictive for posttransplant relapse and survival. ${ }^{92,93}$

In our clinical practice, we utilize patient and disease characteristics coupled with pre- and post-transplant MRD assays as metrics to counsel patients about their risk of relapse. Awaiting further validation, we believe these are useful parameters, especially when conjugated to riskstratified maintenance approaches. Nonetheless, we recommend the use of off-label FLT3-TKIs such as sorafenib because of our favorable experience and the accumulating data with this regard, which led to the EBMT recommendations (Bazarbachi et al, 2020). ${ }^{47}$ HMAs still represent a cornerstone maneuver to upregulate neoantigens and modulate immune responses post-ASCT when used alone or in various upcoming combinations (HMA+ DLI or venetoclax, etc.). One would, however, ask if pretransplant therapy matters in this setting and whether responding favorably or not to azacitidine as initial therapy could affect the outcomes of post-ASCT maintenance. Novel agents such as ADCs and BCL2-inhibitors may provide a favorable approach despite little knowledge about the effect of these molecules on the graft and their potential toxicities. Immune stimulation with agents such as ICPs currently remains investigational awaiting welldesigned clinical trials. Additionally, we must continue to explore the genetic profiling of AML and its ramifications.

\section{Future Challenges and Directions}

Disease relapse remains a paramount endpoint to treating physicians and patients, far beyond the use of survival endpoints alone based on small single-center trials. With the recent surge of therapeutic opportunities, the priority should 
be to tailor randomized trials with refined conditioning regimens to post-transplant strategies while routinely incorporating MRD and genomic assays. This will require a solid partnership between the transplant community, academia and the pharmaceutical institutions for innovative and wellintegrated approaches. A model trial in this setting also needs to assess the activity of a certain approach and its effect on GVHD. There is a steadily increasing number of novel agents, mostly of oral bioavailability, which could be preferred for maintenance therapy owing to their activity, dosing schedules, as well as minimal hematological toxicities. Other areas of interest include the use of MoAbs, ICP inhibitors and possibly products of cellular engineering (vaccines, modified chimeric antigen receptor T-cells, etc.). As a reflection of toxicities, we strongly support the integration of quality-oflife (QoL) metrics and patient-reported outcomes as informative endpoints in the design of these prospective randomized trials.

\section{Disclosure}

The authors report no conflicts of interest in this work.

\section{References}

1. The Cancer Genome Atlas Research Network. Genomic and epigenomic landscapes of adult de novo acute myeloid leukemia. $N$ Engl J Med. 2013;368:2059-2207. doi:10.1056/NEJMoa1301689

2. Döhner H, Estey E, Amadori S, et al. Diagnosis and management of acute myeloid leukemia in adults: recommendations from an international expert panel, on behalf of the European LeukemiaNet. Blood. 2010;115(3):453-474.

3. Burnett A, Russell N, Hills R, et al. A randomized comparison of daunorubicin $90 \mathrm{mg} / \mathrm{m} 2 \mathrm{vs} 60 \mathrm{mg} / \mathrm{m} 2$ in AML induction: results from the UK NCRI AML17 trial in 1206 patients. Blood. 2015;125 (25):3878-3885. doi:10.1182/blood-2015-01-623447

4. Marcucci G, Haferlach T, Döhner H. Molecular genetics of adult acute myeloid leukemia: prognostic and therapeutic implications. $J$ Clin Oncol. 2011;29(5):475-486. doi:10.1200/JCO.2010.30.2554

5. Terwijn M, van Putten W, Kelder A, van der Velden V, Brooimans R, Pabst T. High prognostic impact of flow cytometric minimal residual disease detection in acute myeloid leukemia: data from the HOVON/ SAKK AML 42A study. J Clin Oncol. 2013;31(31):3889-3897. doi:10.1200/JCO.2012.45.9628

6. Ivey A, Hills R, Simpson MA, et al. Assessment of minimal residual disease in standard- risk AML. $N$ Engl J Med. 2016;374:422-433. doi:10.1056/NEJMoa1507471

7. Freeman S, Hills R, Virgo P, et al. Measurable residual disease at induction redefines partial response in acute myeloid leukemia and stratifies outcomes in patients at standard risk without NPM1 mutations. J Clin Oncol. 2018;36(15):1486-1497. doi:10.1200/JCO.2017.76.3425

8. Wingard J, Majhail N, Brazauskas R, et al. Long-term survival and late deaths after allogeneic hematopoietic cell transplantation. J Clin Oncol. 2011;29(16):2230-2239. doi:10.1200/JCO.2010.33.7212

9. Bejanyan N, Weisdorf D, Logan B, et al. Survival of patients with acute myeloid leukemia relapsing after allogeneic hematopoietic cell transplantation: a center for International Blood and Marrow Transplant Research Study. Biol Blood Marrow Transplant. 2015;21 (3):454-459. doi:10.1016/j.bbmt.2014.11.007
10. Ganzel C, Sun Z, Cripe L, et al. Very poor long-term survival in past and more recent studies for relapsed AML patients: the ECOG-ACRIN experience. Am J Hematol. 2018;93:1074-1081. doi:10.1002/ajh.25162

11. Thanarajasingam G, Kim H, Cutler C, et al. Outcome and prognostic factors for patients who relapse after allogeneic hematopoietic stem cell transplantation. Biol Blood Marrow Transplant. 2013;19 (12):1713-1718. doi:10.1016/j.bbmt.2013.09.011

12. Bazarbachi A, Schmid C, Labopin M, et al. Evaluation of trends and prognosis over time in patients with AML relapsing after allogeneic hematopoeitic cell transplant reveals improved survival for young patients in recent years. Clin Cancer Res. 2020b;3134.

13. Craddock C. Pharmacological methods to reduce disease recurrence. Hematology Am Soc Hematol Educ Program. 2013;2013:63-69. doi:10.1182/asheducation-2013.1.63

14. Choi J, Ritchey J, Prior J, et al. In vivo administration of hypomethylating agents mitigate graft-versus host disease without sacrificing graft-versus-leukemia. Blood. 2010;116(1):129-139. doi:10.1182/ blood-2009-12-257253

15. Goodyear O, Dennis M, Jilani N, et al. Azacitidine augments expansion of regulatory $\mathrm{T}$ cells after allogeneic stem cell transplantation in patients with acute myeloid leukemia (AML). Blood. 2012;119 (14):3361-3369. doi:10.1182/blood-2011-09-377044

16. Mathew N, Baumgartner F, Braun L, et al. Sorafenib promotes graft-versus-leukemia activity in mice and humans through IL-15 production in FLT3-ITD-mutant leukemia cells. Nat Med. 2018;24 (3):282-291. doi:10.1038/nm.4484

17. Armand P, Kim H, Zhang M, et al. Classifying cytogenetics in patients with acute myelogenous leukemia in complete remission undergoing allogeneic transplantation: a Center for International Blood and Marrow Transplant Research study. Biol Blood Marrow Transplant. 2012;18(2):280-288. doi:10.1016/j. bbmt.2011.07.024

18. Schlenk R, Döhner K, Krauter J, et al. Mutations and treatment outcome in cytogenetically normal acute myeloid leukemia. $N$ Engl $J$ Med. 2008;358:1909-1918. doi:10.1056/NEJMoa074306

19. Walter R, Buckley S, Pagel J, et al. Significance of minimal residual disease before myeloablative allogeneic hematopoietic cell transplantation for AML in first and second complete remission. Blood. 2013;122(10):1813-1821. doi:10.1182/blood-2013-06-506725

20. Festuccia M, Deeg H, Gooley T, et al. Minimal identifiable disease and the role of conditioning intensity in hematopoietic cell transplantation for myelodysplastic syndrome and acute myelogenous leukemia evolving from myelodysplastic syndrome. Biol Blood Marrow Transplant. 2016;22(7):1227-1233. doi:10.1016/j.bbmt.2016.03.029

21. Milano F, Gooley T, Wood B, et al. Cord blood transplant in patients with minimal residual disease. $N$ Engl J Med. 2016;375:944-953. doi:10.1056/NEJMoa1602074

22. D'Souza A, Lee S, Zhu X, Pasquini M. Current use and trends in hematopoietic cell transplantation in the United States. Biol Blood Marrow Transplant. 2017;23(9):1417-1421. doi:10.1016/j.bbmt.2017.05.035

23. Scott B, Pasquini M, Logan B, et al. Myeloablative versus reduced-intensity hematopoietic cell transplantation for acute myeloid leukemia and myelodysplastic syndromes. J Clin Oncol. 2017;35 (11):1154-1161. doi:10.1200/JCO.2016.70.7091

24. Cruijsen M, Hobo W, van der Velden W, et al. Addition of 10-day decitabine to fludarabine/total body irradiation conditioning is feasible and induces tumor-associated antigen specific T cell responses. Biol Blood Marrow Transplant. 2016;22(6):1000-1008. doi:10.1016/j.bbmt.2016.02.003

25. Sánchez-Abarca L, Gutierrez-Cosio S, Santamaría C, et al. Immunomodulatory effect of 5-azacytidine (5-azaC): potential role in the transplantation setting. Blood. 2010;115(1):107-121. doi:10.1182/blood-2009-03-210393

26. Platzbecker U, Wermke M, Radke J, et al. Azacitidine for treatment of imminent relapse in MDS or AML patients after allogeneic HSCT: results of the RELAZA trial. Leukemia. 2012;26(3):381-389. doi:10.1038/leu.2011.234 
27. Craddock C, Jilani N, Siddique S, et al. Tolerability and clinical activity of posttransplantation Azacitidine in patients allografted for acute myeloid leukemia treated on the RICAZA trial. Biol Blood Marrow Transplant. 2016;22(2):385-390. doi:10.1016/j.bbmt.2015.09.004

28. Guillaume T, Florent Malard F, Leonardo Magro L, et al. Prospective phase II study of prophylactic low-dose azacitidine and donor lymphocyte infusions following allogeneic hematopoietic stem cell transplantation for high-risk acute myeloid leukemia and myelodysplastic syndrome. Bone Marrow Transplant. 2019;54(11):1815-1826. doi:10.1038/s41409-019-0536-y

29. de Lima M, Giralt S, Thall P, et al. Maintenance therapy with low-dose azacitidine after allogeneic hematopoietic stem cell transplantation for recurrent acute myelogenous leukemia or myelodysplastic syndrome. Cancer. 2010;116(23):5420-5431. doi:10.1002/ cncr. 25500

30. El-Cheikh J, Massoud R, Fares E, et al. Low-dose 5-azacytidine as preventive therapy for relapse of AML and MDS following allogeneic HCT. Bone Marrow Transplant. 2017;52(6):918-921. doi:10.1038/bmt.2017.31

31. Oran B, de Lima M, Garcia-Manero G, et al. Maintenance with 5-azacytidine for acute myeloid leukemia and myelodysplastic syndrome patients. Blood. 2018;132(Supplement 1):971. doi:10.1182/ blood-2018-99-111582

32. Platzbecker U, Middeke J, Sockel K, et al. Minimal-residual disease guided treatment with azacitidine in MDS/AML patients at imminent risk of relapse: results of the prospective RELAZA2 trial. Blood. 2017;130(Supplement 1):565.

33. Wei AH, Döhner H, Pocock C, et al. The QUAZAR AML-001 maintenance trial: results of a phase III international, randomized, double-blind, placebo-controlled study of CC-486 (oral formulation of azacitidine) in patients with acute myeloid leukemia (AML) in first remission. Blood. 2019;134(supp12):LBA-3. doi:10.1182/blood-2019-132405

34. de Lima M, Oran B, Champlin R, et al. CC-486 maintenance after stem cell transplantation in patients with acute myeloid leukemia or myelodysplastic syndromes. Biol Blood Marrow Transplant. 2018;24 (10):2017-2024. doi:10.1016/j.bbmt.2018.06.016

35. Pusic I, Choi J, Fiala M, et al. Maintenance therapy with decitabine after allogeneic stem cell transplantation for acute myelogenous leukemia and myelodysplastic syndrome. Bone Marrow Transplant. 2015;21(10):1761-1769. doi:10.1016/j.bbmt.2015.05.026

36. Platzbecker U, Middeke J, Sockel K, et al. Measurable residual disease-guided treatment with azacitidine to prevent haematological relapse in patients with myelodysplastic syndrome and acute myeloid leukaemia (RELAZA2): an open-label, multicentre, Phase 2 trial. Lancet Oncol. 2018;19(12):1668-1679. doi:10.1016/S14702045(18)30580-1

37. Stahl M, Gore S, Vey N, Prebet T. Lost in translation? Ten years of development of histone deacetylase inhibitors in acute myeloid leukemia and myelodysplastic syndromes. Expert Opin Investig Drugs. 2016;25(3):307-317. doi:10.1517/13543784.2016.1146251

38. Bug G, Burchert A, Wagner E-M, et al. Phase I/II study of the deacetylase inhibitor panobinostat after allogeneic stem cell transplantation in patients with high-risk MDS or AML (PANOBEST trial). Leukemia. 2017;31(11):2523-2525. doi:10.1038/leu.2017.242

39. Shen L, Pili R. Class I histone deacetylase inhibition is a novel mechanism to target regulatory $\mathrm{T}$ cells in immunotherapy. Oncoimmunology. 2012;1(6):948-950. doi:10.4161/onci.20306

40. Brunet S, Labopin M, Esteve J, et al. Impact of FLT3 internal tandem duplication on the outcome of related and unrelated hematopoietic transplantation for adult acute myeloid leukemia in first remission: a retrospective analysis. J Clin Oncol. 2012;30(7):735-741. doi:10.1200/JCO.2011.36.9868

41. Kayser S, Dohner K, Krauter J, et al. Impact of allogeneic transplantation from matched related and unrelated donors on clinical outcome in younger adult AML patients with FLT3 internal tandem duplications. Blood. 2010;116(21):909. doi:10.1182/blood.V116.21.909.909
42. Sengsayadeth S, Jagasia M, Engelhardt B, et al. Allo-SCT for high-risk AML-CR1 in the molecular era: impact of FLT3/ITD outweighs the conventional markers. Bone Marrow Transplant. 2012;47 (12):1535-1537. doi:10.1038/bmt.2012.88

43. Deol A, Sengsayadeth S, Ahn K, et al. Does FLT3 mutation impact survival after hematopoietic stem cell transplantation for acute myeloid leukemia? A Center for International Blood and Marrow Transplant Research (CIBMTR) analysis. Cancer. 2016;122 (19):3005-3014. doi:10.1002/cncr.30140

44. Döhner H, Estey E, Grimwade D, et al. Diagnosis and management of AML in adults: 2017 ELN recommendations from an international expert panel. Blood. 2017;129(4):424-447.

45. Sakaguchi M, Yamaguchi H, Najima Y, et al. Prognostic impact of low allelic ratio FLT3-ITD and NPM1 mutation in acute myeloid leukemia. Blood $A d v$. 2018;2(20):2744-2754. doi:10.1182/ bloodadvances.2018020305

46. Straube J, Ling V, Hill G, Lane S. The impact of age, NPM1mut, and FLT3ITD allelic ratio in patients with acute myeloid leukemia. Blood. 2018;131(10):1148-1153. doi:10.1182/blood-2017-09-807438

47. Bazarbachi A, Bug G, Baron F, et al. Clinical practice recommendation on hematopoietic stem cell transplantation for acute myeloid leukemia patients with FLT3-internal tandem duplication: a position statement from the Acute Leukemia Working Party of the European Society for Blood and Marrow. Haematologica. 2020a;105 (6):1507-1516. doi:10.3324/haematol.2019.243410

48. Schuurhuis G, Heuser M, Freeman S, et al. Minimal/measurable residual disease in AML: a consensus document from the European LeukemiaNet MRD Working Party. Blood. 2018;131(12):1275-1291. doi:10.1182/blood-2017-09-801498

49. Antar, A., Kharfan-Dabaja, M, Mahfouz, R, \& Bazarbachi, A. Sorafenib maintenance appears safe and improves clinical outcomes in FLT3-ITD acute myeloid leukemia after allogeneic hematopoietic cell transplantation. Clin Lymphoma Myeloma Leuk. 2015;15 (5):298-302.

50. Borthakur G, Kantarjian H, Farhad Ravandi F, et al. Phase I study of sorafenib in patients with refractory or relapsed acute leukemias. Haematologica. 2011;96(1):62-68. doi:10.3324/haematol.2010.030452

51. Brunner A, Li S, Fathi A, et al. Haematopoietic cell transplantation with and without sorafenib maintenance for patients with FLT3-ITD acute myeloid leukaemia in first complete remission. Br J Haematol. 2016;175(3):496-504. doi:10.1111/bjh.14260

52. Battipaglia G, Ruggeri A, Massoud R, et al. Efficacy and feasibility of sorafenib as a maintenance agent after allogeneic hematopoietic stem cell transplantation for Fms-like tyrosine kinase 3-mutated acute myeloid leukemia. Cancer. 2017;123(15):2867-2874. doi:10.1002/ cncr.30680

53. Battipaglia G, Massoud R, Ahmed S, et al. Efficacy and feasibility of sorafenib as a maintenance agent after allogeneic hematopoietic stem cell transplantation for Fms-like tyrosine kinase 3 mutated acute myeloid leukemia: an update. Clin Lymphoma Myeloma Leuk. 2019;19(8):506-508. doi:10.1016/j.clml.2019.04.004

54. Chen Y, Li S, Lane A, et al. Phase I trial of maintenance sorafenib after allogeneic hematopoietic stem cell transplantation for fms like tyrosine kinase 3 internal tandem duplication acute myeloid leukemia. Biol Blood Marrow Transplant. 2014;20(12):2042-2048. doi:10.1016/j.bbmt.2014.09.007

55. Sammons S, Pratz K, Smith B, Karp J, Emadi A. Sorafenib is tolerable and improves clinical outcomes in patients with FLT3-ITD acute myeloid leukemia prior to stem cell transplant and after relapse post-transplant. Am J Hematol. 2014;89(9):936-938. doi:10.1002/ ajh. 23782

56. Antar A, Kharfan-Dabaja M, Mahfouz R, Bazarbachi A. Sorafenib maintenance appears safe and improves clinical outcomes in FLT3-ITD acute myeloid leukemia after allogeneic hematopoietic cell transplantation. Clin Lymphoma Myeloma Leuk. 2015;15 (5):298-302. doi:10.1016/j.clml.2014.12.005 
57. Pratz K, Rudek M, Smith B, et al. Prospective study of peri-transplant use of sorafenib as remission maintenance for FLT3-ITD patients undergoing allogeneic transplantation. Biol Blood Marrow Transplant. 2020;26(2):300-306. doi:10.1016/j. bbmt.2019.09.023

58. Bazarbachi A, Myriam Labopin M, Battipaglia G, et al. Sorafenib improves survival of FLT3-mutated acute myeloid leukemia in relapse after allogeneic stem cell transplantation: a report of the EBMT Acute Leukemia Working Party. Haematologica. 2019;104 (9):e398-e401. doi:10.3324/haematol.2018.211615

59. Bazarbachi A, Lab M, Battipaglia G, et al. Allogeneic stem cell transplantation for FLT3-mutated acute myeloid leukemia: in vivo T-cell depletion and posttransplant sorafenib maintenance improve survival. A Retrospective Acute Leukemia Working Party-European Society for Blood and Marrow Transplant. Clinical Hematology International. 2019:58-74. doi:10.2991/chi.d.190310.001

60. Burchert A, Bug G, Fritz L, et al. Sorafenib maintenance after allogeneic hematopoietic stem cell transplantation for acute myeloid leukemia with FLT3-internal tandem duplication mutation (SORMAIN). J Clin Oncol. 2020;38(26):2993-3002. doi:10.1200/ JCO.19.03345

61. Xuan L, Wang Y, Huang F, et al. Sorafenib maintenance in patients with FLT3-ITD acute myeloid leukaemia undergoing allogeneic haematopoietic stem-cell transplantation: an open-label, multicentre, randomised phase 3 trial. Lancet Oncol. 2020 Sep;21(9):1201-1212.

62. Stone R, Mandrekar S, Sanford B, Laumann K, Geyer S, Bloomfield C. Midostaurin plus chemotherapy for acute myeloid leukemia with a FLT3 mutation. $N$ Engl J Med. 2017;377:454-464. doi:10.1056/NEJMoa1614359

63. Schlenk R, Weber D, Fiedler W, Salih H, Wulf G, Salwender H. Midostaurin added to chemotherapy and continued single-agent maintenance therapy in acute myeloid leukemia with FLT3-ITD. Blood. 2019;133(8):840-851. doi:10.1182/blood-2018-08-869453

64. Levis M, Brown P, Smith B, Stine A, Pham R, Stone R. Plasma inhibitory activity (PIA): a pharmacodynamic assay reveals insights into the basis for cytotoxic response to FLT3 inhibitors. Blood. 2006;108(10):3477-3483. doi:10.1182/blood-2006-04-015743

65. Strati P, Kantarjian H, Ravandi F, Nazha A, Borthakur G, Daver N. Phase I/II trial of the combination of midostaurin (PKC412) and 5-azacytidine for patients with acute myeloid leukemia and myelodysplastic syndrome. Am J Hematol. 2015;90(4):276-281. doi:10.1002/ajh.23924

66. Maziarz R, Patnaik M, Scott B, Mohan S, Deol A, Rowley S. A phase 2 randomized trial investigating standard of care 6 midostaurin after allogeneic stem cell-transplant in FLT3-ITD-mutated AML. Blood. 2018;132(Supplement 1):662. doi:10.1182/blood2018-99-113582

67. Smith C, Wang Q, Chin C, Salerno S, Damon L, Levis M. Validation of ITD mutations in FLT3 as a therapeutic target in human acute myeloid leukaemia. Nature. 2012;485(7397):260-263. doi:10.1038/ nature 11016

68. Perl A, Altman J, Cortes J, Smith C, Litzow M, Baer M. Selective inhibition of FLT3 by gilteritinib in relapsed or refractory acute myeloid leukaemia: A multicentre, first-in-human, open-label, Phase 1-2 study. Lancet Oncol. 2017;18(8):1061-1075. doi:10.1016/S14702045(17)30416-3

69. Sandmaier B, Khaled S, Betul Oran B, Gammon G, Trone D, Frankfurt O. Results of a phase 1 study of quizartinib (AC220) as maintenance therapy in subjects with acute myeloid leukemia in remission following allogeneic hematopoietic cell transplantation. Blood. 2014;124(21):428. doi:10.1182/blood.V124.21.428.428

70. Smith C, Paguirigan A, Jeschke G, Lin K, Massi E, Theodore Tarver T. Heterogeneous resistance to quizartinib in acute myeloid leukemia revealed by single-cell analysis. Blood. 2017;130(1):48-58. doi:10.1182/blood-2016-04-711820
71. Galanis A, Ma H, Rajkhowa T, Ramachandran A, Small D, Cortes J. Crenolanib is a potent inhibitor of FLT3 with activity against resistance-conferring point mutants. Blood. 2014;123(1):94-100. doi:10.1182/blood-2013-10-529313

72. Heisterkamp N, Jenster G, Hoeve J, Zovich D, Pattengale P, Groffen J. Acute leukaemia in bcr/abl transgenic mice. Nature. 1990;344(6263):251-253. doi:10.1038/344251a0

73. Li L, Piloto O, Nguyen H, Greenberg K, Takamiya K, Racke F. Knock-in of an internal tandem duplication mutation into murine FLT3 confers myeloproliferative disease in a mouse model. Blood. 2008;111(7):3849-3858. doi:10.1182/blood-2007-08109942

74. Jan M, Snyder T, Corces-Zimmerman M, et al. Clonal evolution of preleukemic hematopoietic stem cells precedes human acute myeloid leukemia. Sci Transl Med. 2012;4(149):149ra118. doi:10.1126/ scitranslmed.3004315

75. Roboz G, DiNardo C, Stein E, de Botton S, Mims A, Prince G. Ivosidenib induces deep durable remissions in patients with newly diagnosed IDH1-mutant acute myeloid leukemia. Blood. 2020;135 (7):463-471. doi:10.1182/blood.2019002140

76. Stein E, DiNardo C, Pollyea D, Fathi A, Roboz G, Altman J. Enasidenib in mutant IDH2 relapsed for refractory AML. Blood. 2017;130(6):722-731. doi:10.1182/blood-2017-04-779405

77. Uchida A, Isobe Y, Asano J, Uemura Y, Hoshikawa M, Takagi M. Targeting BCL2 with venetoclax is a promising therapeutic strategy for "double-proteinexpression" lymphoma with MYC and BCL2 rearrangements. Haematologica. 2019;104(7):1417-1421. doi:10.332 4/haematol.2018.204958

78. Pollyea D, Stevens B, Jones C, Winters A, Pei S, Minhajuddin M. Venetoclax with azacitidine disrupts energy metabolism and targets leukemia stem cells in patients with acute myeloid leukemia. Nat Med. 2018;24(12):1859-1866. doi:10.1038/s41591-018-0233-1

79. DiNardo C, Wei A. How I treat acute myeloid leukemia in the era of new drugs. Blood. 2020;135(2):85-96. doi:10.1182/ blood.2019001239

80. Fukushima N, Minami Y, Kakiuchi S, Kuwatsuka Y, Hayakawa F, Jamieson C. Small-molecule Hedgehog inhibitor attenuates the leukemia-initiation potential of acute myeloid leukemia cells. Cancer Sci. 2016;107(10):1422-1429. doi:10.1111/ cas. 13019

81. Terao T, Minami Y. Targeting hedgehog ( $\mathrm{Hh})$ pathway for the acute myeloid leukemia treatment. Cells. 2019;8(4):312. doi:10.3390/ cells 8040312

82. Cortes J, Heidel F, Hellmann A, et al. Randomized comparison of low dose cytarabine with or without glasdegib in patients with newly diagnosed acute myeloid leukemia or high-risk myelodysplastic syndrome. Leukemia. 2019;33(2):379-389. doi:10.1038/s41375-0180312-9

83. Middeke J, Fang M, Cornelissen J, Mohr B, Appelbaum F, Stadler M. Outcome of patients with abnl(17p) acute myeloid leukemia after allogeneic hematopoietic stem cell transplantation. Blood. 2014;123 (19):2960-2967. doi:10.1182/blood-2013-12-544957

84. Sallman D, DeZern A, Garcia-Manero G, Steensma D, Roboz G, Sekeres M. Phase 2 results of APR-246 and azacitidine (AZA) in patients with TP53 mutant myelodysplastic syndromes (MDS) and oligoblastic acute myeloid leukemia (AML). Blood. 2019;134 (Supplement_1):676. doi:10.1182/blood-2019-131055

85. Oshikawa G, Kakihana K, Saito M, Aoki J, Najima Y, Kobayashi T. Post-transplant maintenance therapy with azacitidine and gemtuzumab ozogamicin for high-risk acute myeloid leukaemia. $\mathrm{Br}$ J Haematol. 2015;169(5):756-759. doi:10.1111/bjh.13248

86. Kadia T, Cortes J, Ghorab A, Ravandi F, Jabbour E, Guastad N. Nivolumab (Nivo) maintenance (maint) in high-risk (HR) acute myeloid leukemia (AML) patients. $J$ Clin Oncol. 2018;36 (15_suppl):7014. doi:10.1200/JCO.2018.36.15_suppl.7014 
87. Sockel K, Bornhaeuser M, Mischak-Weissinger E, Trenschel R, Wermke M, Unzicker C. Lenalidomide maintenance after allogeneic HSCT seems to trigger acute graft-versus-host disease in patients with high-risk myelodysplastic syndromes or acute myeloid leukemia and $\operatorname{del}(5 \mathrm{q})$ : results of the LENAMAINT trial. Haematologica. 2012;97(9):e34-5. doi:10.3324/haematol.2012.067629

88. Bashey A, Medina B, Corringham S, Pasek M, Carrier E, Vrooman L. CTLA4 blockade with ipilimumab to treat relapse of malignancy after allogeneic hematopoietic cell transplantation. Blood. 2009;113(7):1581-1588. doi:10.1182/blood-2008-07-168468

89. Davids M, Kim H, Bachireddy P, Costello C, Liguori R, Savell A. Ipilimumab for patients with relapse after allogeneic transplantation. N Engl J Med. 2016;375(2):143-153. doi:10.1056/NEJMoa1601202

90. Albring J, Inselmann S, Sauer T, Schliemann C, Altvater B, Kailayangiri S. PD-1 checkpoint blockade in patients with relapsed AML after allogeneic stem cell transplantation. Bone Marrow Transplant. 2017;52(2):317-320. doi:10.1038/bmt.2016.274
91. Walter R, Gooley T, Wood B, Milano F, Fang M, Sorror M. Impact of pretransplantation minimal residual disease, as detected by multiparametric flow cytometry, on outcome of myeloablative hematopoietic cell transplantation for acute myeloid leukemia. J Clin Oncol. 2011;29(9):1190-1197. doi:10.1200/JCO.2010. 31.8121

92. Thol F, Gabdoulline R, Liebich A, Klement P, Schiller J, Kandziora C. Measurable residual disease monitoring by NGS before allogeneic hematopoietic cell transplantation in AML. Blood. 2018;132(16):1703-1713. doi:10.1182/blood-2018-02829911

93. Kim T, Ho Moon J, Ahn J, Kim Y, Lee S, Ahn S. Next-generation sequencing-based posttransplant monitoring of acute myeloid leukemia identifies patients at high risk of relapse. Blood. 2018;132 (15):1604-1613. doi:10.1182/blood-2018-04-848028

\section{Publish your work in this journal}

The Journal of Blood Medicine is an international, peer-reviewed, open access, online journal publishing laboratory, experimental and clinical aspects of all aspect pertaining to blood based medicine including but not limited to: Transfusion Medicine; Blood collection, Donor issues, Transmittable diseases, and Blood banking logistics; Immunohematology; Artificial and alternative blood based therapeutics; Hematology; Biotechnology/nanotechnology of blood related medicine; Legal aspects of blood medicine; Historical perspectives. The manuscript management system is completely online and includes a very quick and fair peer-review system. Visit http://www.dovepress.com/testimonials.php to read real quotes from published authors. 